Imperial/TP/1-02/17

hep-th/0203015

\title{
Revisiting Supergravity and Super Yang-Mills Renormalization
}

\author{
K.S. Stelle \\ Department of Physics, Imperial College, London SW7 2BW, UK
}

\begin{abstract}
Standard superspace Feynman diagram rules give one estimate of the onset of ultraviolet divergences in supergravity and super Yang-Mills theories. Newer techniques motivated by string theory but which also make essential use of unitarity cutting rules give another in certain cases. We trace the difference to the treatment of higher-dimensional gauge invariance in supersymmetric theories that can be dimensionally oxidized to pure supersymmetric gauge theories.
\end{abstract}

\section{NON-RENORMALIZATION THEOREMS}

The problem we shall consider in this article $^{1}$ is to reconcile the predictions for the onset of ultraviolet divergences in supergravity and super Yang-Mills theories [1]made from traditional superspace Feynman diagram analysis and those made on the basis of unitarity and factorization implied by embedding in string theory [10]. The question of ultraviolet divergences is one of the oldest concerns in the subject of quantum gravity. Although the problem of order-by-order perturbative finiteness has been solved in superstring theory, the question remains of interest because superstring theory acts as a physical regulator for its limiting supergravity theory, so the orders at which various operators become relevant to supergravity divergences gives information about the presence of the same operator with a finite coefficient in superstring theory.

Many problems of current interest involve the scaling behavior of quantum corrections, which is determined by how strongly they are renormalized. And the verification of duality conjectures makes detailed use of supergravity radiative corrections. And a number of mysteries have arisen: some correlation functions appear to be mysteriously "protected" for reasons that are not yet understood. So, in this context it is important to get to the bottom of any disagreements between different viewpoints on the ultraviolet problem.

One good way to approach the study of non-renormalization theorems is through the background-field method. A classic example of a non-renormalization theorem is the Adler-Bardeen theorem [2]. This may be understood from the standpoint of the background field method [3] as follows. Consider the Yang-Mills Chern-Simons operator

\footnotetext{
${ }^{1}$ This article is based upon work done in collaboration with P.S. Howe and M. Petrini.
} 
under a background-quantum split $A_{a}^{r}=\mathcal{B}_{a}^{r}+Q_{a}^{r}$ :

$$
K^{a}=4 g^{2} \varepsilon^{a b c d}\left[A_{b}^{r}\left(\partial_{c} A_{d}^{r}+\frac{1}{3} g f^{r s t} A_{c}^{s} A_{d}^{t}\right)\right] .
$$

This operator has the property that its divergence gives the Pontryagin density: $\partial_{a} K^{a}=$ $2 g^{2} F_{a b}^{r}{ }^{*} F^{r a b}$, but it is not itself gauge invariant. It's renormalization properties, however, are important in the context of anomalies. When one expands it in powers of the quantum field $Q_{a}^{r}$, the lowest term is just the expression (1) with $\mathcal{B}_{a}^{r}$ substituted for $A_{a}^{r}$, so it is no more background gauge invariant than (1). Nor is the term linear in $Q_{a}^{r}$ invariant under background gauge transformations. But the term quadratic in $Q_{a}^{r}$ can be written $4 g^{2} \varepsilon^{a b c d} Q_{a}^{r} D_{b}(\mathcal{B}) Q_{c}^{r}$, which is fully background gauge invariant. Similarly, the term cubic in $Q_{a}^{r}$ is background invariant, since $Q_{a}^{r}$ transforms as a tensor under the background transformations.

The above structure for $K^{a}$ has the striking consequence that although $K^{a}$ itself is not gauge invariant, the terms actually used in calculating one-particle-irreducible (1PI) Feynman diagrams with operator insertions of $K^{a}$ are actually background gauge invariant. Accordingly, the renormalization of this operator can only be through gauge invariant operators, of which class it is not itself a member. Consequently, neither $K^{a}$ itself nor does any gauge-invariant operator $O$ mix with it under renormalization, and so for the anomalous dimensions one has $\gamma_{K K}=\gamma_{O K}=0$.

The Adler-Bardeen theorem then arises as follows. In the presence of axial anomalies, the axial current $j_{a}^{5}$ is not conserved; one has the famous relation $\partial_{a} J^{5 a}=2 g^{2} c F_{a b}^{r}{ }^{*} F^{r a b}$, where $c$ is the anomaly coefficient, first occurring at the one-loop level. However, there is a non-gauge-invariant current $j_{a}^{\prime 5}=J_{a}^{5}-c K_{a}$ that is conserved. Since conserved operators are not renormalized, one has the renormalization group equation $\mu \frac{\partial}{\partial \mu} j_{a}^{\prime 5}=0$. In consequence, and taking into account the $K^{a}$ anomalous dimensions from above, $\gamma_{K K}=\gamma_{j K}=0$ together with independence of the operators $j_{a}^{5}$ and $K_{a}$, one finds

$$
\begin{aligned}
\gamma_{j j}-c \gamma_{K j} & =0 \\
\mu \frac{\partial c}{\partial \mu} & =0 ;
\end{aligned}
$$

and (3) is just the Adler-Bardeen theorem.

We may draw from this brief review the moral that "vestigial" consequences of gauge invariance, such as those revealed by the above use of the background field method, can give rise to important non-renormalization properties, such as the Adler-Bardeen theorem.

Now let us review the basic non-renormalization theorem [4] of $N=1$ supersymmetry, also from the point of view of the background field method. We consider the basic Wess-Zumino model, based upon a chiral superfield $\phi ; \bar{D}_{\dot{\alpha}} \phi=0$. In the background field method, one again makes a background-quantum split, $\phi=\varphi+Q$. The background superfield $\varphi$ then appears only on the external legs of 1PI Feynman diagrams used in calculating the effective action $\Gamma(\varphi)$, while the quantum field $Q$ occurs on the inside lines of diagrams.

The derivation of the Feynman rules for the $Q$ lines may be carried out either taking particular care with the functional delta functions arising from variational derivatives 
with respect to chiral superfields, or one may solve the chirality constraint for $Q$ and work henceforth with unconstrained general scalar superfields. Adopting the second approach, one writes $Q=\bar{D}^{2} X$, where $\bar{D}^{2}=\bar{D}^{\dot{\alpha}} \bar{D}_{\dot{\alpha}}$. Expanding then the action into background and quantum fields, one finds that all terms except for the $O\left(X^{0}\right)$ terms can now be re-written as integrals over the full superspace. Thus, e.g., a mass term decomposes as $m \int d^{4} x d^{2} \theta \phi^{2} \longrightarrow m \int d^{4} x d^{2} \theta \varphi^{2}+2 m \int d^{4} x d^{4} \theta \varphi X+\int d^{4} x d^{4} \theta X \bar{D}^{2} X$.

The vertices and propagators for the quantum $X$ fields used in 1PI diagrams are derived from terms containing 2 or more $Q$ fields, expanded as above and written as full superspace integrals. On the other hand, the background $\varphi$ superfields continue to appear always as constrained, chiral, superfields. Hence the non-renormalization theorem for $D=4, N=1$ supersymmetry arises: perfectly supersymmetric invariants such as chiral superspace integrals over prepotentials may occur in the original action of the theory, but they may not occur as counterterms. From the structure of the Feynman rules, all quantum corrections to the effective action $\Gamma$ must be written as full superspace integrals. Thus, chiral superspace integrals like $\int d^{4} x d^{2} \theta f(\phi)$ are not renormalized. Note that one cannot try to frustrate the strictures of this non-renormalization theorem by writing chiral superspace integrals in forms like $\int d^{4} x d^{4} \theta \phi \square^{-1} D^{2} \phi$, because the operator $\square^{-1}$ is spatially non-local, and this then is not in accord with the requirements of Weinberg's theorem [5] on the locality of counterterms.

For extended supersymmetries that can be given a complete linear realization in superspace, similar results are found. In particular, for matter fields such as the $N=2$ hypermultiplet, one directly obtains a similar result: hypermultiplet self-interactions are not renormalized [6].

For gauge multiplets and supergravity multiplets, there is a further subtlety, which affects however only the one-loop diagrams. As in the case of the $N=1$ chiral multiplet, one introduces prepotentials for the quantum fields that appear on internal lines of diagrams, while background fields appear through constrained superfields, the original superspace gauge connections. The procedure of gauge fixing and introduction of the ghost actions, however, introduces some complications. Superspace gauge symmetry parameters are described by constrained superfields, e.g. the chiral gauge superfield $\Lambda$ for $N=1, D=4$ super Yang-Mills theory, which is constrained in the background field method by a background-covariant constraint $\overline{\mathcal{D}}_{\dot{\alpha}}(\mathcal{B}) \Lambda=0$. In the construction of the corresponding ghost action for a chiral ghost superfield written with the chirality constraint solved via the introduction of a prepotential, e.g. $\overline{\mathcal{D}}^{2} S$, one finds that $S$ has its own gauge invariance $\delta S=\overline{\mathcal{D}}^{\dot{\alpha}} \bar{\Lambda}_{\dot{\alpha}}$. This requires a new gauge fixing and ghost action, which in turn has a new gauge invariance with chiral parameter $\bar{\Lambda}_{\dot{\alpha} \dot{\beta}}$. This process continues indefinitely, with further gauge parameters $\bar{\Lambda}_{\dot{\alpha} \dot{\beta} \dot{\gamma}}$, etc, and these will all couple to the background fields unless one cuts off the interactions with the background at some order. This necessitates the introduction of a background gauge field prepotential, thus breaking the non-renormalization theorem, but only for the one-loop diagrams. 


\section{DIVERGENCE ESTIMATES FOR EXTENDED SUPERSYMMETRY}

Theories with extended supersymmetry, and in particular the maximal supergravity and super Yang-Mills theories, face another problem in estimating the onset of ultraviolet divergences: not all of a given theory's supersymmetry can be linearly realized "offshell," i.e. with an algebra that closes without use of the equations of motion. This is an old problem in supersymmetric theories, whose full implications are still to this day not clear. In analyzing the possibilities for infinities, one can choose between several alternatives for the "next best thing." For example, one can see what is the maximal degree of supersymmetry that can be linearly realized off-shell and then use that, keeping at the same time full Lorentz invariance [7]. Or, alternatively, one can keep the full automorphism symmetry that appears in the on-shell formalism without auxiliary fields but sacrifice Lorentz invariance, e.g. through use of a light-cone formalism [8]. Similar conclusions are obtained in either formalism; however, the Lorentz-covariant approach has been used to study more cases. The upshot is that for $N=4 \leftrightarrow 3,2,1$ super YangMills one can use formalisms with $M=2,2,1$ off-shell supersymmetry. For $N=8 \leftrightarrow$ $7,6,5,4,3,2,1$ supergravity theories one can use formalisms with $M=4,3,3,2,2,2,1$ off-shell supersymmetry.

Putting all of this together, one has a general supersymmetric non-renormalization theorem: For gauge and supergravity multiplets at loops $\ell \geq 2$, and for matter multiplets at all loop orders, counterterms must be written as full superspace integrals for the maximal off-shell linearly realizable supersymmetry, with background gauge invariant integrands written without using prepotentials for the background fields.

Although the full power of a given theory's supersymmetry cannot always be used to determine the structure of counterterms from supersymmetric Ward identities - only the linearly realized supersymmetry gives useable Ward identities in general - the full supersymmetry can still play a rôle. Things simplify greatly if one considers the counterterms subject to the classical field equations. For the first non-vanishing one of these, the Ward identities for the full (nonlinear) supersymmetry requires full supersymmetry invariance, subject to the imposition of the classical field equations [9]. There is no requirement, however, on being able to write the counterterm as a full superspace integral for the full nonlinear supersymmetry. Nonetheless, this requirement does serve to rule out some counterterms that would by themselves be acceptable according to the above theorem, but whose coefficients become linked via the full supersymmetry to the coefficients of terms disallowed by the above theorem.

Of course, the above considerations cannot say exactly when the first divergences can occur in a given theory - the most they can say is up to which loop orders the divergences cannot. Nonetheless, in the study of divergences one has become used to the maxim that if something isn't ruled out, it will occur. So any further cancellations would appear "miraculous" from the standpoint of the above analysis. Here is a table of the expectations for divergences in maximal (i.e. $N=1$ in $D=10$ or $N=4$ in $D=4$ ) super Yang-Mills theory:

and in maximal (i.e. $N=1$ in $D=11$ or $N=8$ in $D=4$ ) supergravity: 
TABLE 1. Super Yang-Mills divergence expectations, standard Feynman rules.

\begin{tabular}{|l|c|c|c|c|c|c|}
\hline Dimension & 10 & 8 & 7 & 6 & 5 & 4 \\
loop $L$ & 1 & 1 & 2 & 3 & 4 & $\infty$ \\
gen. form & $\partial^{2} F^{4}$ & $F^{4}$ & $\partial^{2} F^{4}$ & $\partial^{2} F^{4}$ & $F^{4}$ & finite \\
\hline
\end{tabular}

TABLE 2. Supergravity divergence expectations, standard Feynman rules.

\begin{tabular}{|l|c|c|c|c|c|c|c|}
\hline Dimension & 11 & 10 & 8 & 7 & 6 & 5 & 4 \\
loop $L$ & 2 & 1 & 1 & 2 & 2 & 2 & 3 \\
gen. form & $\partial^{6} R^{4}$ & $\partial^{2} R^{4}$ & $R^{4}$ & $\partial^{4} R^{4}$ & $\partial^{2} R^{4}$ & $R^{4}$ & $R^{4}$ \\
\hline
\end{tabular}

\section{STRING-INSPIRED CALCULATIONS VIA CUTTING RULES}

Traditional Feynman diagram techniques quickly become unmanageable. For example, a 5-loop diagram in $N=8, D=4$ supergravity may have on the order of $10^{30}$ terms in its algebraic expression. So a better procedure is clearly desirable.

Just such a procedure (let us call it the $\mathrm{BD}^{2} \mathrm{PR}$ procedure [10]) has been offered in recent years, using factorization properties of supergravity amplitudes inherited from string theory, coupled with a procedure for extracting the ultraviolet divergences from the absorptive parts of amplitudes, using the cutting rules. The key elements of this procedure are:

1) the optical theorem

2) dimensional regularization

Specifically, from the optical theorem, one determines the imaginary part of a oneloop amplitude in terms of a product of tree amplitudes, for example. One might worry that by this procedure one looses information about the real parts of amplitudes. But this is avoided by a careful use of dimensional regularization. The optical theorem works best for amplitudes containing logarithms, where one can clearly see the relation between real and imaginary parts, e.g. $\ln (s)=\ln (|s|)+\mathrm{i} \pi \theta(s)$. This can be enhanced by dimensional regularization, which produces many additional logarithms, e.g. $s^{-2 \varepsilon}=1-2 \varepsilon \ln (s)+\ldots$, where $\varepsilon=D-D_{\text {physical }}$; these help to determine amplitudes providing one calculates diagrams factorized using the cutting rules to all powers in $\varepsilon$. The remaining essentials in the $\mathrm{BD}^{2} \mathrm{PR}$ procedure are

3) field-theory amplitudes result from string amplitudes in the $\alpha^{\prime} \rightarrow 0$ limit

4) the KLT relations [11] between closed and open string tree-level S-matrices.

As a combined example of the two last points, one has a relation between supergravity and super Yang-Mills 4-point amplitudes, arising from the $\alpha^{\prime} \rightarrow 0$ limit of superstring theory, where $M_{4}^{\text {tree }}$ is a supergravity amplitude and $A_{4}^{\text {tree }}$ is a SYM amplitude:

$$
M_{4}^{\text {tree }}(1,2,3,4)=-\mathrm{i} s_{12} A_{4}^{\text {tree }}(1,2,3,4) A_{4}^{\text {tree }}(1,2,3,4) .
$$


Using the KLT relations, one may organize supergravity amplitudes and super YangMills amplitudes to display a squaring relationship. Moreover, amplitude calculations with irreducible supermultiplets reduce to scalar amplitudes multiplied by index-bearing factors. This is illustrated in Fig. 1.
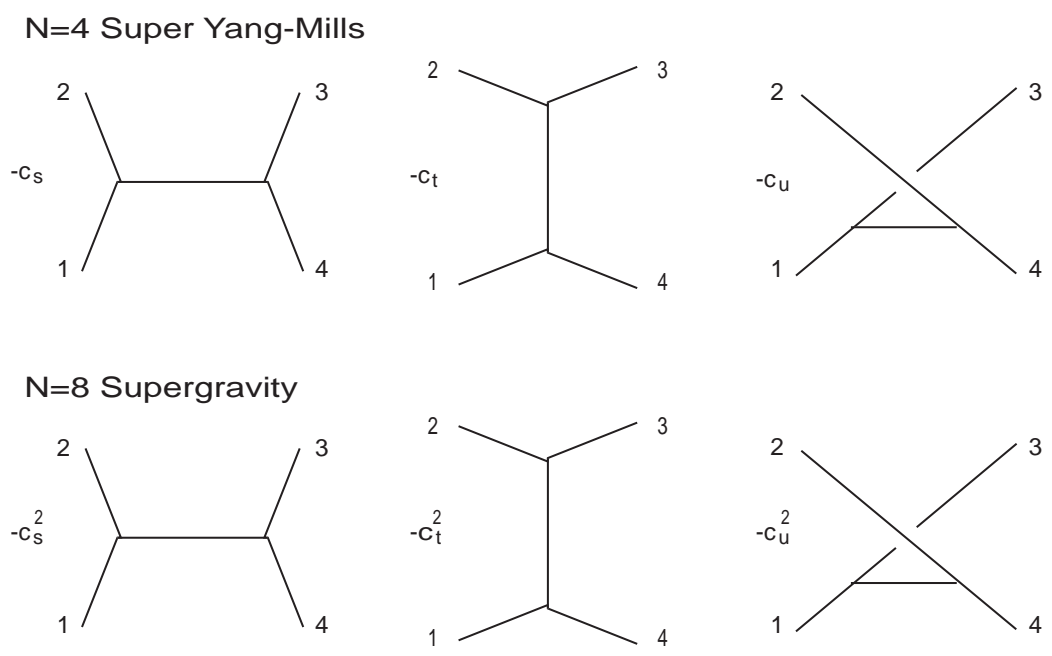

FIGURE 1. Tree-level color-ordered $N=4$ SYM and $N=8$ SG amplitudes expressed in terms of $\phi^{3}$ diagrams. Supergravity coefficients are squares of the SYM coefficients.

The relations between supergravity and super Yang-Mills amplitudes continues on to higher loops, enabling SG and SYM amplitudes to be calculated in terms of scalare diagrams. A one-loop example is illustrated in Fig. 2

$$
\mathrm{N}=4 \mathrm{SYM} \quad \mathrm{N}=8 \mathrm{SG}
$$

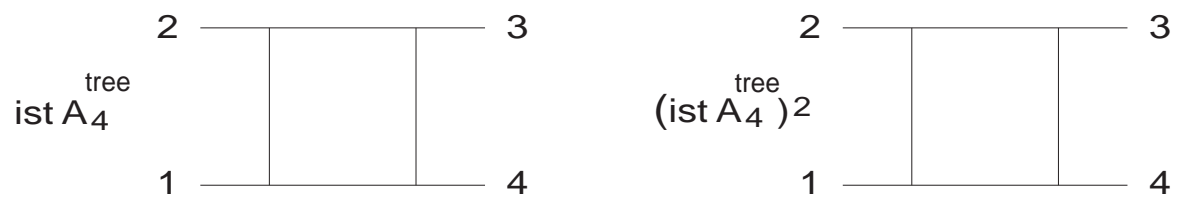

FIGURE 2. Relation between $N=4 \mathrm{SYM}$ theory and $N=8 \mathrm{SG}$ amplitudes at the one-loop level. Tree amplitudes multiply scalar field loop diagrams.

Using these techniques, $\mathrm{BD}^{2} \mathrm{PR}$ obtain the following predictions for the first on-shell divergences in the maximal super Yang Mills theories:

TABLE 3. Maximal super Yang-Mills divergence expectations from cutting rules.

\begin{tabular}{|l|c|c|c|c|c|c|}
\hline Dimension & 10 & 8 & 7 & 6 & 5 & 4 \\
loop $L$ & 1 & 1 & 2 & 3 & 6 & $\infty$ \\
gen. form & $\partial^{2} F^{4}$ & $F^{4}$ & $\partial^{2} F^{4}$ & $\partial^{2} F^{4}$ & $\partial^{2} F^{4}$ & finite \\
\hline
\end{tabular}

and in maximal supergravity they obtain: 
TABLE 4. Maximal supergravity divergence expectations from cutting rules.

\begin{tabular}{|l|c|c|c|c|c|c|c|}
\hline Dimension & 11 & 10 & 8 & 7 & 6 & 5 & 4 \\
loop $L$ & 2 & 1 & 1 & 2 & 3 & 4 & 5 \\
gen. form & $\partial^{6} R^{4}$ & $\partial^{2} R^{4}$ & $R^{4}$ & $\partial^{4} R^{4}$ & $\partial^{6} R^{4}$ & $\partial^{6} R^{4}$ & $\partial^{4} R^{4}$ \\
\hline
\end{tabular}

So one can see that the $\mathrm{BD}^{2} \mathrm{PR}$ results appear to be predicting a "miracle" in the onset of divergences in the maximal SYM and SG theories. One may summarize their results for the two maximal theories by the rule that the maximal SYM theories are finite for $D<\frac{6}{L}+4(L>1)$ and the maximal supergravity theories are finite for $D<\frac{10}{L}+2$ $(L>1)$. A striking aspect of this improvement is that it applies only to the maximal supersymmetric theories. The cutting-rule analysis of $N \leq 6$ supergravity in $D=4$ shows the onset of divergences to be exactly as predicted on the basis of standard Feynman diagram analysis, equal to those summarized for the maximal theories in tables 1 and 2 .

Of course, there is strictly speaking no firm contradiction here, for the standard Feynman diagram analysis gave only a lower bound on the possible loop order for the first on shell surviving infinities. Nonetheless, the difference is striking. The $\mathrm{BD}^{2} \mathrm{PR}$ results are in fact more than an estimate. They give an actual calculation of the coefficients of the various divergences, limited only in that for loops $L>2$ only two-particle cuts have been considered; a full calculation would require general $m$ particle cuts.

\section{COUNTERTERM ANALYSIS}

The study of counterterms for super Yang-Mills and supergravity theories has been going on since the discovery of these theories. Pure Einstein gravity diverges at the two-loop order with an $R^{3}$ counterterm, as found by explicit calculations [12]. In supergravity theories, on-shell supersymmetry rules out all on-shell surviving counterterms at the $L=1$ and $L=2$ loop orders. In particular, this dramatically causes the cancellation of the $R^{3}$ counterterm that is present in pure Einstein gravity.

The first dangerous supergravity counterterm occurs at $L=3$ loops in $D=4$, and has an $R^{4}$ generic structure. This counterterm involves the curvature through the square of the Bel-Robinson tensor

$$
T_{\mu v \rho \sigma}=R_{\mu \alpha v \beta} R_{\rho}^{\alpha}{ }_{\rho}^{\beta}+{ }^{*} R_{\mu \alpha \nu \beta}{ }^{*} R^{\alpha}{ }_{\rho}^{\beta} \sigma .
$$

This tensor is a kind of analogue of the stress tensor for gravity: it is totally symmetric and totally traceless (for pure gravity) and it is covariantly conserved on any index. In an analogy to the supersymmetric $T_{\mu v} T^{\mu v}$ counterterms that exist for matter systems in a gravitational background, pure supergravity has a $T_{\mu v \rho \sigma} T^{\mu \nu \rho \sigma}+$ fermionic counterterm [13], appropriate by standard power counting for the $L=3$ order of perturbation theory. The existence of similar counterterms was also found for all $D=4$ extended supergravities $[14,15,16]$. These same expressions occur as important corrections with finite coefficients to superstring theories; the question in that case is also at which loop order they first appear. 
Consider now in some more detail the structure of the $R^{4}$ counterterm for the higher $N \geq 4$ supergravities in $D=4$ [16]. The on-shell supergravity multiplet is described by a superfield $W_{[i j k l]}$ carrying automorphism group $\mathrm{SU}(N)$ indices and satisfying the full on-shell superspace constraints

$$
\begin{aligned}
D_{\alpha}^{i} W_{j k l m} & =\frac{-4}{N-3} \delta_{[j}^{i} D_{\alpha}^{n} W_{k l m] n} \\
\bar{D}_{\dot{\alpha} i} W_{j k l m} & =\bar{D}_{\dot{\alpha}[i} W_{j k l m]} .
\end{aligned}
$$

At the linearized level, which all that we need concern ourselves with here, each component of $W_{i j k l}$ is a field $F$ with $2 s$ symmetrized spinor indices of the same chirality. The constraints imply $\partial_{\dot{\alpha}}^{\alpha_{1}} F_{\alpha_{1} \cdots \alpha_{2 s}}=\square F_{\alpha_{1} \cdots \alpha_{2 s}}=0$. These on-shell component field strengths describe massless spin $s$ for $s \leq 2$, including $N(N-1)(N-2)(N-3) / 12 s=0$ scalars for $N \leq 7$, and 70 scalars for the self-conjugate $N=8$ theory. All of these scalars arise from dimensional reduction of the metric and the higher-dimensional gauge field $A_{\mu v \rho}$.

The $R^{3}$ counterterm is written for the $N=8$ theory as an on-shell superspace integral over a subset of 16 out of the 32 fermionic coordinates. We recall that the nonrenormalization theorem requires that this counterterm (which is the first one nonvanishing on shell) be fully supersymmetric under the full (in general nonlinear, although here we consider only the linearized level) $N=8$ supersymmetry, but that it also be possible to be expressed in off-shell $N=4$ superfields in which no prepotentials are introduced and the counterterm is written as a full superspace integral. The $\int d^{16} \theta$ structure of the $R^{4}$ counterterm allows this to happen. In linearized superspace, the structure of the on-shell counterterm is [16]

$$
\Delta \Gamma=\kappa^{4} \int d^{4} x\left(d^{16} \theta\right)_{232848}\left(W^{4}\right)_{232848}
$$

where the 232848 representation of $\mathrm{SU}(8)$ is described by the $4 \times 4$ square Young tableau with four rows of four boxes each. One can also view this as a superinvariant built from the 232848 rep. extracted from the quadratic product of two $\left(W^{2}\right) 1764$ superfields, where the 1764 rep. is described by two columns of 4 boxes. This is the multiplet that contains the Bel-Robinson tensor, which in 2-component indices is $T_{\alpha \dot{\alpha}, \beta \dot{\beta}, \gamma \dot{\gamma}, \delta \dot{\delta}}=C_{\alpha \beta \gamma \delta} C_{\dot{\alpha} \dot{\beta} \dot{\gamma} \dot{\delta}}$, where $C_{\alpha \beta \gamma \delta}$ is the Weyl tensor in two-component notation. Since this counterterm satisfies all the requirements of the non-renormalization theorem discussed earlier.

\section{GAUGE INVARIANCE AND THE DISCREPANCY IN DIVERGENCE ESTIMATES}

It is striking that the standard Feynman diagram analysis and the $\mathrm{BD}^{2} \mathrm{PR}$ analysis agree in almost all cases, with the only disagreements coming for the maximal super YangMills and supergravity theories. This provides a clue to what might be going on. Again, it seems to come down to a difference in the treatment of gauge invariance. Both of the theories for which there is a discrepancy have the property that they can be dimensionally oxidized to theories in which all the scalar fields have disappeared into components of 
higher-dimensional gauge fields, metrics, and so on. In particular, the $D=4, N=8$ supergravity theory oxidizes to $D=11, N=1$ supergravity, in which all fields are gauge fields, including all the spinors.

This observation implies that the origin of the difference in divergence estimates lies in the way gauge invariance is being treated. Indeed, if one adopts the rule that background fields must appear in the counterterm in a way that can also be oxidized to a gauge-invariant structure in the higher dimension, then the two divergence estimates agree [17]. This also works for the super Yang-Mills theories.

Another example that shows how the discrepancy between the two divergence estimates is removed by the revised rule for gauge invariance occurs in $D=5$ maximal super Yang-Mills theory. This has 5 scalars, but all of them are absorbed upon oxidization of the theory to $D=10, N=1$ super Yang-Mills theory. Another example is $D=5$, $N=1 \mathrm{SYM}$, which has a single scalar. This can, however, be absorbed into the gauge field upon oxidization to $D=6, N=1 \mathrm{SYM}$ theory.

This leaves us with a question, on which we are going to end this review of divergence problems and their attendant mysteries. Are there circumstances in which higherdimensional gauge symmetries may still effectively govern the structure of counterterms in lower-dimensional theories? If so, then the standard superspace Feynman rules and the $\mathrm{BD}^{2} \mathrm{PR}$ cutting-rule analysis give the same results. But how this comes about remains mysterious. There does not appear to be any direct way to preserve more of the higherdimensional gauge symmetries than that which is manifest in the lower dimension upon dimensional reduction. The gauge transformations depending upon the compactified dimensions are the ones that would have to impart structure to the counterterms, but these are lost when one suppresses dependence upon the compactified coordinates, or sets to zero the corresponding loop momenta.

So we end with this question: do supersymmetry and the vestiges of superstring theory present in supersymmetric gauge theories conspire to keep alive the higher gauge symmetries? These are the most unified theories that we know, and it just might be that in whatever dimension one formulates them, they remember their higher-dimensional origins.

\section{REFERENCES}

1. P.S. Howe, and K.S. Stelle, Int. J. Mod. Phys.A4 (1989) 1871.

2. S. Adler and W.A. Bardeen, Phys. Rev. 182 (1969) 1517.

3. P. Breitenlohner, D. Maison and K.S. Stelle, Phys. Lett. 134B (1984) 63.

4. M.T. Grisaru, W. Siegel and M. Roček, Nucl. Phys. B159, 429 (1979).

5. S. Weinberg, Phys. Rev. 118 (1959) 838.

6. P.S. Howe, K.S. Stelle and P.K. Townsend, Nucl. Phys. B214 (1983) 519.

7. P.S. Howe, K.S. Stelle and P.K. Townsend, Nucl. Phys. B236 (1984) 125.

8. S. Mandelstam, Nucl. Phys. B213 (1983) 149;

L. Brink, O. Lindgren and B. Nilsson, Phys. Lett. 123B (1983) 328.

9. P.S. Howe and K.S. Stelle, Phys. Lett. 137B (1984) 175.

10. Z. Bern, L. Dixon, D. Dunbar, M. Perelstein and J. Rosowsky, Nucl. Phys. B530 (1998) 401.

11. H. Kawai, D,C. Lewellyn and S.-H.H. Tye, Nucl. Phys. B269 (1986) 1.

12. M.H. Goroff and A. Sagnotti, Nucl. Phys. B266 (1986) 709;

A.E.M. van de Ven, Nucl. Phys. B378 (1992) 309. 
13. S. Deser, J. H. Kay and K.S. Stelle, Phys. Rev. Lett. 38 (1977) 527.

14. S. Deser and J. H. Kay, Phys. Lett. 76B (1978) 400.

15. R.E. Kallosh, Phys. Lett. 99B (1981) 122.

16. P.S. Howe, K.S. Stelle and P.K. Townsend, Nucl. Phys. B191 (1981) 445.

17. P.S. Howe, M. Petrini and K.S. Stelle, in preparation. 